\title{
BIBECHANA
}

A Multidisciplinary Journal of Science, Technology and Mathematics

ISSN 2091-0762 (online)

Journal homepage: http://nepjol.info/index.php/BIBECHANA

\section{Experimental investigation on anisotropic surface properties of crystalline silicon}

\author{
Shobha Kanta Lamichhane \\ Department of physics, Prithvi Narayan Campus, Pokhara, Nepal \\ E-mail: sklamichhane@hotmail.com \\ Article history: Received 31 May, 2011; Accepted 25 July, 2011
}

\section{Abstract}

Anisotropic etching of silicon has been studied by wet potassium hydroxide $(\mathrm{KOH})$ etchant with its variation of temperature and concentration. Results presented here are temperature dependent etch rate along the crystallographic orientations. The etching rate of the (111) surface family is of prime importance for microfabrication. However, the experimental values of the corresponding etch rate are often scattered and the etching mechanism of (111) remains unclear. Etching and activation energy are found to be consistently favorable with the thermal agitation for a given crystal plane. Study demonstrate that the contribution of microscopic activation energy that effectively controls the etching process. Such a strong anisotropy in $\mathrm{KOH}$ allows us a precious control of lateral dimensions of the silicon microstructure.

Keywords: microfabrication; activation energy; concentration; anisotropy; crystal plane

\section{Introduction}

Anisotropic etching of silicon is used to obtain varied microstructures [1-3], and anisotropic etchants extensively used are $\mathrm{KOH}$, EDP (ethylene diamine, pyrocatechol), TMH (tetryl methylammonium hydroxide), etc. The mechanisms of anisotropic etching of silicon and the etching processes that occur in the crystalline surfaces are not well known. Many authors have suggested a different sort of mechanism for the reactions to explain the anisotropic behaviour of etching of silicon in alkaline solutions [4, 5]. Seidel et al [6] has proposed an electro-chemical model which involves the transfer of the electrons from ions in the solution to the crystal surface. In this model, the rate determining step is the transfer of electrons from the surface state, which lies in the forbidden gap, to the conduction band. This process is thermally activated. Seidel states that the difference in energy between final and initial state contributes significantly to the macroscopic activation energy. Anisotropy can be accounted for by the fact that the energy level of the surface state depends on the surface orientation. Thus the (111) surface which exhibits the highest activation energy when etched with $\mathrm{KOH}$, has the lowest level energy state. The (110) and (100) planes present roughly the same level below that corresponding to (111) surface state. In other words, they have approximately the same activation energy. On the other hand, Elwenspoek [7] puts forward a model inspired from the crystal growth theories in which the etch rate should be governed by a nucleation barrier of one atomic layer deep cavities and the activation energy depends on the step-free energy and under saturation. 
Micro-electro mechanical structure (MEMS) devices are the output of either an etching process (or micromachining) from larger structures or are built up atom by atom through material deposition processes.

Maintaining dimensional control and retaining consistent mechanical property is considerably difficult because feature sizes are in the micrometer range. Silicon MEMS offer these challenges. Formation of a functional MEMS structure on a substrate, it is necessary to etch the thin films previously deposited or the substrate itself. In general there are two type of etching process: wet etching, where the material is dissolved when immersed in a chemical solution and, dry etching, where the material is sputtered or dissolved using reactive ions. In the following we briefly discuss the most popular technology, wet etching, is a simplest etching technology. All it requires is a container with a liquid solution that will dissolve the material. Unfortunately, there are complications since a mask is desired to selectively etch the material. One must find a mask that will not dissolve or at least etch much slower then the material to be patterned. Anisotropic is contrast to isotropic etching means different etch rates in different plane surfaces. The classic example of this is the (111) crystal plane sidewalls that appears when etching a hole in (100) silicon wafer in a chemical such as $\mathrm{KOH}$. The result is a pyramid shaped whole instead of a hole with round sidewalls with an isotropic etchant.

Etching technologies in thin film processes play an important role in the semiconductor industry. Wet chemical etching techniques are used extensively in semiconductor processing because of their low cost, high throughput, and excellent selectivity. Important progress in the fabrication of microelectrical structure with integrated circuits has been achieved. Though detailed kinetic and electrochemical measurements have not been made, $\mathrm{KOH}$ solutions are extensively used for the etching of single crystal silicon [8, 9]. Fabrication of UMOS transistors on silicon (111) wafers for high power and high current densities has been achieved by applying $\mathrm{KOH}$ anisotropic wet etching to the silicon substrate. Other applications of $\mathrm{KOH}$ wet etching include the fabrication of VMOSFETs, radio frequency amplifiers, power supplies, and microcomputers [10]. Using its selective and anisotropic etching properties, $\mathrm{KOH}$ wet etching has also been applied to yield such devices as field emission devices, optical waveguides, pressure sensors and ink nozzles [11]. $\mathrm{KOH}$ wet etching is one of the old anisotropic orientation dependent wet etching techniques. Etching of (100) oriented silicon using aqueous $\mathrm{KOH}$ creates $\mathrm{V}$-shaped grooves with (111) planes at an angle of $54.74^{\circ}$ from the (100) surface. $\mathrm{KOH}$ solution can also be used to produce mesa structures. There are several models proposed for the silicon etching mechanism in aqueous $\mathrm{KOH}$. Bean and Runyan found that the slow etch rate in the (111) direction is a consequence of the diamond lattice structure because the (111) plane is a double layer bound together by more atomic bonds than that of other planes. Glembocki et al. proposed an electrochemical reaction mechanism that exists in the rate determining step for $\mathrm{KOH}$ etching. A compromise model was proposed by Seidel et al. in 1990, assuming that electrochemical reactions dominate during etching and that anisotropic etching was caused by the orientation-dependent number of dangling bonds available per surface area. The (100) silicon surface etches faster than the (111) because the free surface of (100) silicon is attacked by one $\mathrm{OH}$ per silicon atom, whereas the (111) silicon surface is attacked by two $\mathrm{OH}$ per silicon atoms [12]. Although the mechanisms are not clear and need to be answer all these questions. The approach followed so as to determine the significant factor in a given electrochemical process and their quantification are prime importance from technological point of view. Such proposed quires are being addressed in this work. If we have answer of all these quires in hand, then there is no doubt of etching silicon in aqueous $\mathrm{KOH}$ is of both technological and fundamental importance.

Etching of (100) oriented silicon using aqueous $\mathrm{KOH}$ creates $\mathrm{V}$-shaped grooves with (111) planes at an angle of $54.74^{\circ}$ from the (100) surface. $\mathrm{KOH}$ solution can also be used to produce mesa structures. There are several models proposed for the silicon etching mechanism in aqueous $\mathrm{KOH}$. Bean and Runyan found that the slow etch rate in the (111) direction is a consequence of 
the diamond lattice structure because the (111) plane is a double layer bound together by more atomic bonds than that of other planes [10]. A compromise model was proposed by assuming electrochemical reactions dominate during etching and that anisotropic etching was caused by the orientation-dependent number of dangling bonds available per surface area. The (100) $\mathrm{Si}$ surface etches faster than the (111) because the free surface of (100) Si is attacked by one $\mathrm{OH}$ per $\mathrm{Si}$ atom, whereas the (111) $\mathrm{Si}$ surface is attacked by two OH per Si atoms. Although the mechanisms are not clear, it is known that electrochemical reactions play an important role in this process.

\section{Methodology}

The design of the present work was lab based experimental work. The procedure followed during this study was mentioned herewith sequentially. The wafers used in this study were thermally bonded silicon on insulator (SOI) wafers with (100) orientation. The thickness of the top silicon, buried $\mathrm{SiO}_{2}$ layer and bottom silicon were 2 and $450 \mathrm{~mm}$ respectively. The wafers were of $p$-type silicon with a resistivity of $4-631022 \mathrm{~V}-\mathrm{cm}$ for the top silicon and $12-15 \mathrm{~V} \mathrm{~cm}$ for the bottom silicon. Only the top silicon layer was etched by the $\mathrm{KOH}$ solution. First, the SOI wafers were prepared with standard RCA cleaning. A $450 \AA$ layer of $\mathrm{Si}_{3} \mathrm{~N}_{4}$, which acts as a $\mathrm{KOH}$ etching mask, was deposited on each wafer using low pressure chemical vapor deposition (LPCVD). Oxide can be used as an etch mask for short periods in the $\mathrm{KOH}$ solution, but for long periods, nitride is a better etch mask as it etches more slowly. The SOI wafer was then cut into $10 \mathrm{~mm} \times 10 \mathrm{~mm}$ pieces. With the diced pieces held on a vacuum chuck, positive photoresist, OCG 825 [Sutter et al, 2003; pp 4] was used to pattern the $\mathrm{Si}_{3} \mathrm{~N}_{4}$ for the $\mathrm{KOH}$ etching mask. The nitride was etched with CF4 plasma and then the exposed portion of the top silicon on each SOI wafer was etched in $\mathrm{KOH}$ solutions of varying temperature and concentration. To assure the samples were free of particulate and other airborne contaminants. The experiments were conducted in a clean room environment.

\section{Results and Discussion}

$\mathrm{KOH}$ etch is a bulk silicon etch whose etch rate is depends on the orientation of the crystal plane can be exploited to create specific geometries (for example, V-grooves sown below). Formation of such geometrical structure on silicon crystal depends on various factors: temperature, concentration, activation energy, surface finish and its cleaning process etc. We have performed on crystalline silicon with aqueous $\mathrm{KOH}$ for the temperature ranging from $70^{\circ} \mathrm{C}$ to $90^{\circ} \mathrm{C}$ and its concentration was $30 \%$. Our results shows that the optimum etch rate with the minimum surface roughness, indicates low activation energy at $80^{\circ} \mathrm{C}$. With this one can easily understand that a single result is the outcome of various factors including $\mathrm{KOH}$ concentration.

The etch rate versus temperature as well as etch rate versus $\mathrm{KOH}$ concentration are displayed in Figure 2. Figure 2 (a) and (b) shows that etch rate increases with increasing reaction temperature, regardless about $\mathrm{KOH}$ concentration. Within the working range investigated, the etch rate exhibits a maximum at approximately $20 \% \mathrm{KOH}$ and $90^{\circ} \mathrm{C}$. Below $70^{\circ} \mathrm{C}$, little dependence of etch rate with the $\mathrm{KOH}$ concentration is observed. Without considering the effect of etching, we conclude that the highest etch rate can be achieved with a high temperature reaction. Figure 2 (d) demonstrate the effect of changing $\mathrm{KOH}$ concentration with the etch rate. As we conclude from figure. 2 that ER depends on concentration are less significant than reaction temperature.

Dimensional control of a microstructure is strongly related to MEMS performance. Etching technique allows control of the dimension. Working in micromachining we need to both add and 
remove the material in a controlled way. Adding material on a silicon wafer can be done by chemical vapor deposition (CVD). Removing material is done almost by chemical etching, where a material is removed atom by atom [13]. This can be done either in a liquid (wet etching) or in plasma (dry etching). Still the most simple and versatile anisotropic wet chemical etching for silicon is in $\mathrm{KOH}$. Generally, etch anisotropy is given by,

$$
E_{A}=1-\left(E R_{L} / E R_{V}\right) \text {, }
$$

where $E R_{L}$ and $E R_{V}$ are the lateral and vertical etch rates. A process is said to be purely anisotropic $\left(E_{A}=1\right)$ if lateral etch rate is zero. On the other hand, if $E_{A}=0$, lateral and vertical etch rates are identical and etching is said to be isotropic. The geometry what we got is shown in Fig.1 below.

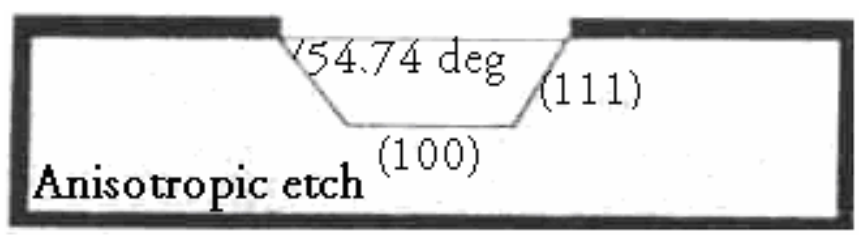

Fig.1: Schematic showing anisotropic etches of silicon

The final shape of the etched wafer depends highly on relative etching speed along crystallographic planes. The (111) plane is comparatively inert to alkaline etchants, while the relative etching speed of other planes depends on nature of etchant, temperature, concentration etc. In our experiment, we have taken $33 \% \mathrm{KOH}$ by weight as an etchant for silicon (111) and (100) planes ambient conditions, unless otherwise specified.

\subsection{Surface finish of Etched Silicon}

Contaminants are attached to the wafer surface physically or chemically. Chemical cleaning is necessary in many steps during fabrication. The process that is widely used is so called "RCA cleaning" consist of two consecutive cleaning solutions including $\mathrm{H}_{2} \mathrm{O}-\mathrm{H}_{2} \mathrm{O}_{2}-\mathrm{NH}_{4} \mathrm{OH}$ (standard clean1, i.e. SC1 clean) and $\mathrm{H}_{2} \mathrm{O}-\mathrm{H}_{2} \mathrm{O}_{2}-\mathrm{HCL}$ (standard clean 2, i.e. SC2). The SC1 cleaning with volume ratios typically 5:1:1 is to remove organic contaminants. The SC1 can remove particles physically attached to a wafer surface by etching with $\mathrm{NH}_{4} \mathrm{OH}$ that detaches the particles from the wafer surface and prevents re-deposition. The SC2 clean, with typical volume ratios of 6:1:1 removes metals from wafer surface and prevents re-deposition [13]. A modified RCA clean (i.e., dilute HF after SC1) is designed to eliminate the thin oxide layer grown on silicon surface. Modification of RCA clean exists on the volume ratios. As for example, in order to reduce silicon surface roughness one needs to greatly reduce the volume fraction of $\mathrm{NH}_{4} \mathrm{OH}$ in SC1 from the original composition. Surface taken by AFM is depicted in fig. 3 below. Other cleaning processes, such as piranha $\left(\mathrm{H}_{2} \mathrm{SO}_{4}-\mathrm{H}_{2} \mathrm{O}_{2}-\mathrm{H}_{2} \mathrm{O}\right)$, ozonized water etc. are also effective. However, RCA clean have been most popular cleaning process, is adopted in this work. 


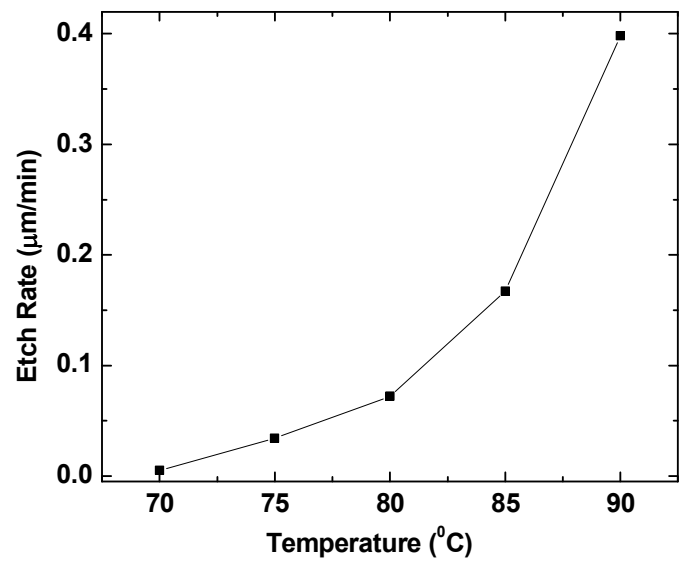

(a)

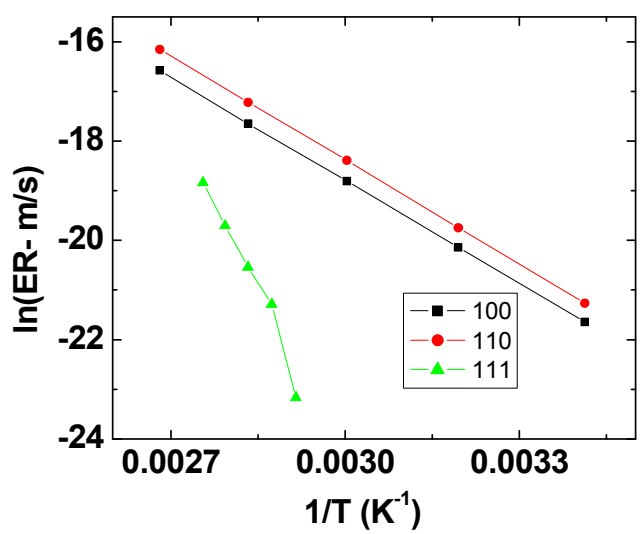

(c)

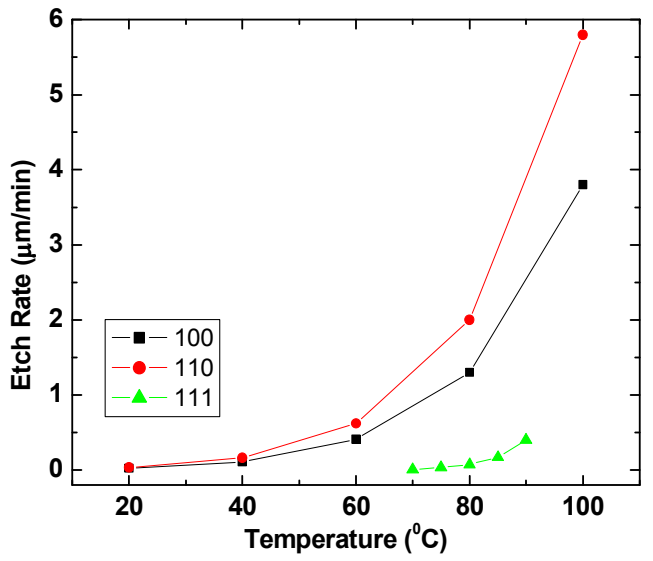

(b)

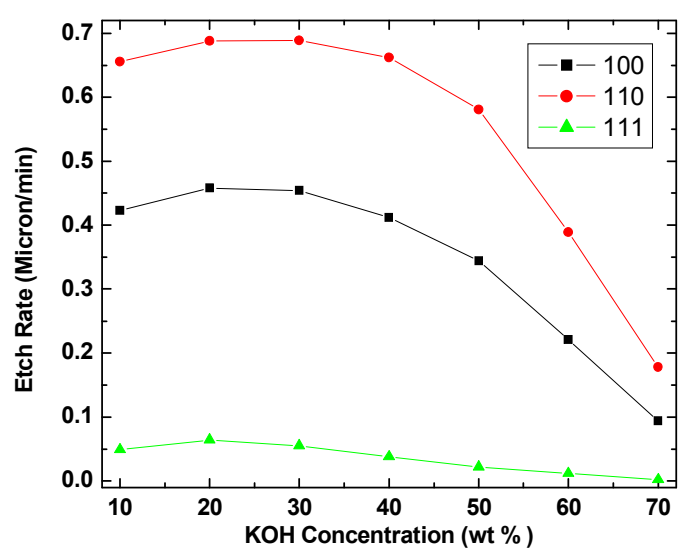

(d)

Fig. 2: Behaviors of etching of crystalline silicon:

(a) Variation of ER along (111) plane with temperature

(b) Variation of ER along $(111,110,100)$ plane with temperature

(c) Arrhenius plot of ER for primary crystal planes

(d) Variation of ER along $(111,110,100)$ plane with $\mathrm{KOH}$ concentration

\subsection{Association between Etch Rate and Activation Energy}

The macroscopic activation energy of ER is explained by the sum of two terms. One corresponds to the average of the microscopic activation energies and other accounts for the existence of fluctuation in the fraction of particles at a fixed temperature. ER depends on $p_{i}$ and $T$. In order to understand this point, we define $E R$ as

$$
E R=\frac{\Delta Z_{c . m .}}{\Delta t}=\frac{1}{\Delta t} \sum_{i}\left(\Delta Z_{c . m .}\right)_{i .} p_{i}
$$

where, $\Delta Z_{c . m}$ is the distance traveled by the center of mass of the surface, $p_{i}$, is the removal probability of the surface atom $i$, and is given by

$$
p_{i}=p_{0 i} e^{-\frac{E_{a i}}{k_{B} T}}
$$


where $T$ is the temperature, $k_{B}$ is the Boltzmann constant, $E_{a i}$ is the microscopic activation energy for removal of site $i$ and $p_{o i}$ is a prefactor. Hence, macroscopic and microscopic activation energy and temperature are interrelated.

Thus, ER is the distance traveled by the moving surface per unit time. When the etching process has reached steady state, ER is simply the ratio of the distance traveled by the center of mass $(\mathrm{CM})$ of the surface $(\mathrm{ZCM})$ to the period of time elapsed $(\mathrm{t})$. i.e. $\mathrm{ER}=\mathrm{ZCM} / \mathrm{t}$. During a modeling, ZCM can be determined as the sum of all individual shifts $(Z C M)_{i}$ of the surface following each successful event, i.e. the sum over all successful particle removal is occurring during ' $t$ '. Although $(Z C M)_{i}$ is typically positive, occasionally it may be negative if the removal of site i involves a reduction in the total number of surface sites [14]. In particular, certain site- types of our formula for wet chemical etching, typically contribute to the motion of the CM with a negative shift on average. Alternatively, we may consider the sum over all events, independently of whether the event is a successful removal or not. Note that the positive/negative shift of the CM of the surface due to the removal of atom i, (ZCM)i, may be calculated independently, whether the atom is removed or not. Equation suggests that the etch rate is composed of two factors: one purely geometrical (the CM-shifts) and one purely numerical. Indeed, ER is proportional to the average number of surface atoms removed from the surface per unit time. Here, proportionality constant $(Z)$ is precisely a measure of the average shifts in the CM of the surface per removed atom. $Z$ is an exclusively geometrical feature of the ER. In particular, it is independent of temperature. Although Z may take different values for different surface orientations, $Z$ does not depend on temperature for a specified orientation. This is an important observation because the appearance of negative CM-shifts (ZCM) may be accepted as the interpretation of the relative importance (weight) of the different particle types for the calculation of an average. Actually, the interpretation becomes meaningless if some of the weights are negative. However, the use of $i$ is free of these artifacts and allows unambiguous interpretation, as shown in this study. There are three alternate ways to determine the rate of removal of particles [15]: (i) as in the case of the ER, of the removal of particles can be determined using only successful events. Note that the averages of the number of surface atoms $\mathrm{N}$ are not constant. (ii) The rate of removal of particles can be written in terms of a sum over the different types of surface sites. The rate of removal of particles may also be expressed in terms of the average fraction of particles removed per unit time. Here we have defined as the average fraction of particles. Activation energy, in relation to the ER, the activation energy (Ea) is, by definition, the slope of the curve.

\subsection{Etched Crystallographic Planes}

It is essential to monitor the evolution of the surface configuration and roughness during etching. Although we are dealing with ideal conditions, this gives us an idea of the difference in etching between the different planes. It is thought that all faces that are rough in the atomic scale etch in the same manner. This is supported by the AFM micrograph representation of one plane viz. (111). This is a characteristic feature of rough faces at the atomic level when etched with an alkaline solution. 

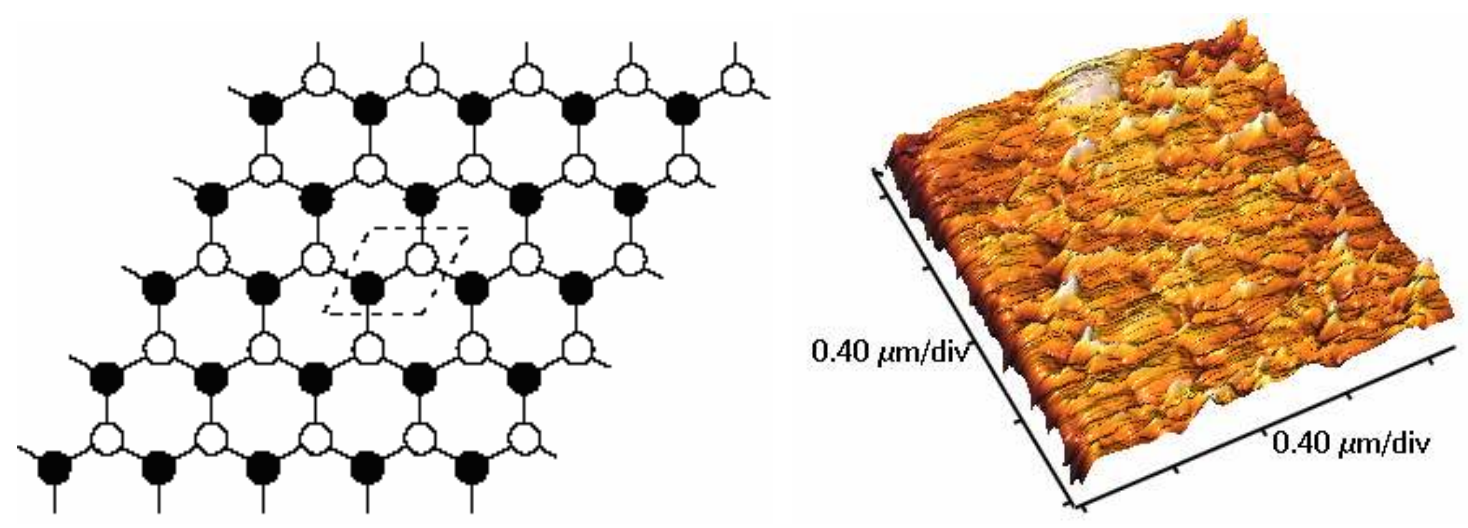

Fig.3: Structure of (111) silicon plane as seen from the top and its AFM micrograph.

Seidel et al [6] has carried out precise experiments to determine the values of the ER of the three silicon orientations (100), (110) and (111). In their experiments, the concentration of $\mathrm{KOH}$ was also modified. The result shows that the anisotropic ratio $(110) /(100)$ has a small dependence on the concentrations (from 10-60\%), and the value is on the order of 1.55 at $80^{\circ} \mathrm{C}$. The macroscopic activation energies were found to be similar for (100) and (110) planes [16], being approximately $0.6 \mathrm{eV}$. We found an anisotropic ratio $(110) /(100)$ of the same order as above (1.5). For activation energies, we found approximately $0.6 \mathrm{eV}$ for (100) and (110) planes.

\section{Conclusion}

The etching mechanism is 'layer-by-layer' removal. This mechanism leads to a particular evolution of the roughness which shows an alternation of peaks. Anisotropic etching of silicon with $\mathrm{KOH}$ is a well-known method of forming grooves in the silicon-surface. Etching of (100) silicon on silicon on insulator wafers was carried out over a wide range of temperatures and concentrations. Using statistical methods, we show that the factors important in the silicon ER are reaction temperature, $\mathrm{KOH}$ concentration, and interaction between them. The activation energy belongs to etching at different $\mathrm{KOH}$ concentration was calculated from Arrhenius plots. In addition, in this work we report a qualitative study of the surface roughness formed in $\mathrm{KOH}$ etching. The smoothness increases with temperature to a critical point and then decreases at very high temperature and concentration. Results presented here are temperature dependent ER along the crystallographic orientations. Based on this work, temperature is a crucial factor, which control ER. Activation and cohesive energies along the crystallographic planes are thus microscopic quantities that determine ER. ER is slow for (111) face and fast in (110). Strong ER anisotropy allows us a precious control of lateral dimension of the microstructure bound by the (111) surface and activation energy depends on the step-free energy and undersaturation.

\section{References}

[1] K. E. Petersen IEEE Proc. 70 (1982) 420.

[2] D. L. Kendall, Annual Rev. Mater. Sci. vol 9, ed R A Huggins (1979) 373.

[3] H. Namatsu, M. Nagase, K. Kurihara, K. Iwadate and K.Murase, Microelectron. Eng. 27 (1995) 7. 
[4] O. J. Glembocki, E D Palik, G R de Guel and D L Kendall, J. Electrochem. Soc. 138 (1991) 1055.

[5] E. D. Palik, V M Bermudez and O J Glembocki, J. Electrochem. Soc. 132 (1985) 871.

[6] H. Seidel, L. Csepregi, A Heuberger and H Baumgartel, J. Electrochem. Soc 137 (1990) 3612.

[7] E. Vazonyi and T. Szkufcik, Journal of Micromechanics and Microengineering 13 (2003) 165.

[8] N. P. Krivorotov, Y. G. Svinolupov, T. I Izaak, V. V. Bychkov, Sensors and Actuatiors A 113(2004) 350.

[9] K.. Mylvaganam and C. L. Zhang, Key engineering materials 233 (2003) 615.

[10] P. Sutter, E. Sutter and M. G. Lagally, Surface Science 30 (2003)1.

[11] P. Waish, R. Omeitchenko, R. K. Kalla, A. Nakano and P. Vashishta, Applied physics letters $82(2003) 118$.

[12] X. Yan, T. Xu, G. Chen, S. Yang, and Huiwenliu, Journal of Physics D: Applied Physics 37 (2004) 907.

[13] P. J.French and A. G. R. Evans, Solid State Electronics 32 (1989) 1.

[14] P. J French. and A.G.R Evans, Journal of Physics E: Scientific Instruments 19 (1986) 1055.

[15] J. Duck Lee, B. Chang Shim, H. Soo and B. Gook, Electron Device Letters 20 (1999) 215.

[16] K. E. Bean and W. R. Runyan, Semiconductor Integrated Circuit Processing Technology, Addision- Wesley, New York 1990. 